\title{
Schistosoma mansoni Heat Shock Protein 70 Elicits an Early Humoral Immune Response in S. mansoni Infected Baboons
}

\author{
Herminia Y Kanamura/ ${ }^{+}$, Kathy Hancock*, Vanderlei Rodrigues**, Raymond T Damian***
}

\begin{abstract}
Departamento de Biologia, Universidade de Taubaté, Av. Tiradentes 500, 12030-010 Taubaté, SP, Brasil and Faculdade de Ciências Farmacêuticas, Universidade de São Paulo, São Paulo, SP, Brasil *Division of Parasitic Diseases, Centers for Diseases Control and Prevention, Atlanta, GA, USA **Faculdade de Medicina de Ribeirão Preto, Universidade de São Paulo, Ribeirão Preto, SP, Brasil ***Department of Cellular Biology, University of Georgia, Athens, GA, USA
\end{abstract}

A study was undertaken to search for DNA recombinant Schistosoma mansoni proteins responsible for eliciting an antibody response from the host at a very early phase after infection. A S. mansoni adult worm cDNA expression library was screened using pooled sera from baboons with four weeks of infection. Based on their specific reactivity with the S. mansoni infected sera and no reactivity when tested against the pre-infection sera from the same baboons, four clones were selected for further studies. Sequence analysis revealed that they were homologous to the S. mansoni heat shock protein 70 (hsp70). The insert sizes of the four selected clones varied from 1150 to $2006 \mathrm{bp}$. The preliminary characterization for antibody reactivity against a panel of baboon sera showed that the longest clone was the most reactive, eight out of eight acute and three out of four chronic sera reacting positively to this clone. The shortest clone was the least reactive. Our results suggest that the S. mansoni hsp70 elicits an early and strong antibody response in baboons and that antibodies to this protein can be detected in chronically infected animals. Therefore S. mansoni hsp70 may be a valid target for immunodiagnosis. However further studies are needed to identify the portion of the hsp70 that best fits the requirements for a valuable diagnostic antigen.

Key words: Schistosoma mansoni - cDNA - heat shock protein 70 - antibody response - baboon

Schistosomiasis continues to be one of the most important and widespread parasitic diseases, despite the great efforts dedicated to eradicating it through drug treatment, control of the snails, health education and sanitary measures. It is still a serious public health problem in more than 70 countries, where, from 600 million at risk, about 200 million people are estimated to be infected (Chitsulo et al. 2000, Gibodat \& Bergquist 2000, WHO 2001). Thus, much research has been done to identify and characterize antigens that may have vaccine and/or diagnostic potential. The application of the recombinant DNA techniques to the studies of Schistosoma antigens has provided some remarkable findings, with definitive characterization of many different antigens (Bergquist 1995, Hamilton et al. 1998, Davis 2000). The humoral immune response to $S$. mansoni has been shown to be directed against a great number of egg, larval and adult stage antigens of the parasite. Among them, a $70 \mathrm{kDa}$ protein molecule was shown to be one of the most immunogenic antigens in S. mansoni. Sera of S. mansoni infected humans and animals, and also mice vaccinated with irradiated cercariae, uniformly rec-

This work received financial support from Fapesp, the Fogarty International Center/NIH, and the UNDP/World Bank/WHO Special Programme for Research and Training in Tropical Diseases

${ }^{+}$Corresponding author. Fax: +55-12-232.7660. E-mail: kanamura@usp.br

Received 28 August 2001

Accepted 19 February 2002 ognized this antigen, identified as a $70 \mathrm{kDa}$ heat shock protein (hsp70) (Hedstrom et al. 1987, 1988). Members of the hsp70 family of proteins have been extensively studied, since hsp70 proteins are one of the most abundant family of proteins expressed by all living organisms, both eukaryotic and prokaryotic (Kaufmann 1990, Mosely 2000). In spite of its high sequence conservation, the hsp70 has been shown to be an immunodominant target of the humoral and cellular response in infections caused by different bacterial, fungal, and parasite pathogens (Davenport et al. 1992, Kakeya et al. 1999, Oliveira-Ferreira et al. 1999, Rico et al. 1999).

Different authors have suggested the use of recombinant S. mansoni hsp70 (Smhsp70) for immunodiagnostic purposes (Hedstrom at al. 1988, Newport et al.1988, Moser at al. 1990), but not much work has been done to determine the frequency of anti-Smhsp70 antibodies in healthy individuals and in patients with autoimmune diseases. Also there is a need to better identify the epitopes of the hsp70 specifically recognized by the host. In the present paper, we report the results of a preliminary characterization of the antibody reactivities demonstrated by four clones isolated from a $S$. mansoni cDNA expression library screened with a pool of sera from baboons in a very early phase of $S$. mansoni infection. This aspect can be important to further characterize the hsp70 as a valid target for an early diagnosis of schistosomiasis. The size of the inserts and the nucleotide sequence of the clones showed that they are coding for different segments of the Smhsp70. The results obtained against a panel of sera from baboons with acute and chronic schistosomiasis suggest different antibody reactivities of the proteins expressed by the four isolated hsp70 clones. 


\section{MATERIALS AND METHODS}

Baboon sera - Seven naive baboons (Papio cynocephalus) were infected with about $350 \mathrm{~S}$. mansoni cercariae per kg of body weight. Serum and stool samples were collected weekly until the 12th week postinfection. All of the baboons excreted $S$. mansoni eggs, beginning between 38 and 41 days after infection, and all harbored from 1,600 to 3,900 adult worms upon autopsy. These baboons, with acute infections, were identified as AC-1 to AC-7, and screened for antibody activity against $S$. mansoni adult worm microsomal antigen (MAMA). Based on the MAMA-FAST-ELISA (Hancock \& Tsang 1986) results, 4-week infection serum pools were formed and used to screen the cDNA expression library. Nine to 11 week postinfection sera were used for the secondary and tertiary rounds of plaque purification of the positive clones from the initial screen.

Additional S. mansoni infected baboon sera were used for preliminary evaluation of the positive clones. Serum from another acutely infected baboon (AC-8), collected at 13 weeks postinfection, and serum from four chronically infected baboons were tested for antibody reactivity with the positive clones. The chronic infection sera were collected from baboons with long lasting S. mansoni infection: CR-1 and CR-2, 5 years; CR-3, 8 years; and CR4, 10 years. The baboons CR-2, CR-3, and CR-4 were treated with praziquantel, respectively 4,6 and 8 years after infection. However, they were not completely cured, as determined by the presence of $S$. mansoni eggs in the feces, 3 to 6 months after treatment. The first baboon CR1 was not treated.

Screening of cDNA library - A S. mansoni adult worm cDNA expression library, constructed in the vector lambda UniZap XR (Stratagene, La Jolla, CA), was screened using standard methods (Sambrook et al. 1989), as already described (Kanamura \& Hancock 1997).

For the primary screening, equal volumes of 4-week postinfection sera from baboons AC-1, AC-2, AC-4, and AC-5 were pooled and used to screen the library. The serum pool was diluted 1:50 with PBS, $0.3 \%$ Nonidet P40, $5 \%$ nonfat dry milk, $5 \mathrm{mg} / \mathrm{ml}$ Escherichia coli lysate, 1 $\mathrm{mM}$ Pefabloc (Boehringer-Mannheim, Indianapolis, IN), 1 $\mu \mathrm{g} / \mathrm{ml}$ leupeptin (Calbiochem, San Diego, CA), and $1 \mu \mathrm{g} /$ $\mathrm{ml}$ pepstatin A (Boehringer-Mannheim). The lysate was prepared from an overnight culture of XL1-Blue E. coli cells. The cells were washed with PBS then lysed in 0.05 M Hepes, pH 7.2, $0.5 \mathrm{M} \mathrm{NaCl}, 0.5 \%$ Nonidet P40, 0.1\% $\mathrm{NaN}_{3}$, and protease inhibitors, as above, by sonication. All sera used for screening or evaluating plaques were absorbed with E. coli lysate and diluted as above. Bound antibodies were detected with goat anti-human immunoglobulin labeled with peroxidase, followed by $\mathrm{H}_{2} \mathrm{O}_{2}$ at $0.003 \%$ and $3,3^{\prime}$-diaminobenzidine at $0.5 \mathrm{mg} / \mathrm{ml}$. Sera and conjugate incubations were for $3 \mathrm{~h}$ at room temperature or overnight at $4^{\circ} \mathrm{C}$. After each incubation, the membranes were washed extensively with PBS, $0.3 \%$ Tween 20 . Then, the membranes were thoroughly washed with PBS, and incubated with substrate for $10 \mathrm{~min}$ at room temperature.

For the secondary and tertiary screening, a pool of 9 , 10 , and 11-week postinfection sera was used. At the ter- tiary screening, it became difficult to identify positive plaques without a surrounding background of negative plaques. To overcome this problem, we included on the agar plate, with the antibody reactive positive clone that we were plaque purifying, a non schistosome clone. A lambda UniZap XR phage clone isolated from a Cryptosporidium parvum cDNA library and coding for C. parvum elongation factor 1-alpha was used to provide the surrounding background of negative plaques. We refer to this method as the immunoenzymatic plaque expression assay (Kanamura \& Hancock 1997). At the tertiary screening, the antibody reactive positive clones were also examined with a pool of preinfection sera from baboons AC-1, AC-2, AC-4, and AC-5, with the $C$. parvum clone as a negative background clones. Clones that reacted positively with the preinfection pool were discarded. Well-isolated positive plaques, reactive only with the infection serum pool, were selected from the tertiary screening and plated again, without the negative background, before they were amplified.

The amplified clones were evaluated for antibody reactivity, by means of the immunoenzymatic plaque expression assay, with a panel of $S$. mansoni infected baboon sera: acute and chronic infection sera, and serial samples from an acute infection. Like the tertiary screening, these clones were plated with the $C$. parvum elongation factor 1-alpha clone providing the negative clone background.

DNA sequencing - All phage clones positive with the early infection sera pool and negative with the preinfection sera pool were DNA sequenced. The insert DNA was amplified using T3 and T7 primers and AmpliTaq polymerase (Perkin-Elmer, Foster City, CA), then purified using a Qiaquick column (Qiagen Inc., Chatsworth, CA). Sequencing was by dye terminator cycle sequencing with AmpliTaq polymerase FS (Perkin-Elmer) followed by polyacrylamide gel electrophoresis using the ABI PRISM 377 DNA sequencer (Perkin-Elmer). Clones were analyzed using the Sequence Navigator software and identified by a BLAST search of GenBank with the computation performed at the National Center for Biotechnology Information using the BLAST network service.

\section{RESULTS}

The acutely infected baboons first showed an antibody response to MAMA at three weeks postinfection (Fig. 1B). At four weeks postinfection, low levels of antiMAMA antibodies could be detected in five of the seven baboons. Four of these baboons, AC-1, AC-2, AC-4, and AC-5, were selected to form the serum pool for screening the cDNA library. Baboons AC- 3 and AC- 6 were excluded because of their low level of antibody response at 4 weeks. Baboon AC-7 was excluded because of an atypical pattern of antibody reactivity. Antibody reactivity patterns of the seven baboons are presented on Fig. 1A, B.

Based on their positive reactivity with the $S$. mansoni infected baboon serum pools, and negative reactivity with the preinfection serum pool, four clones were selected and DNA sequenced. All were identified as identical to the previously reported Smhsp70 gene, accession \#L02415 (Neumann et al. 1992). The gene is on an EcoRI restric- 

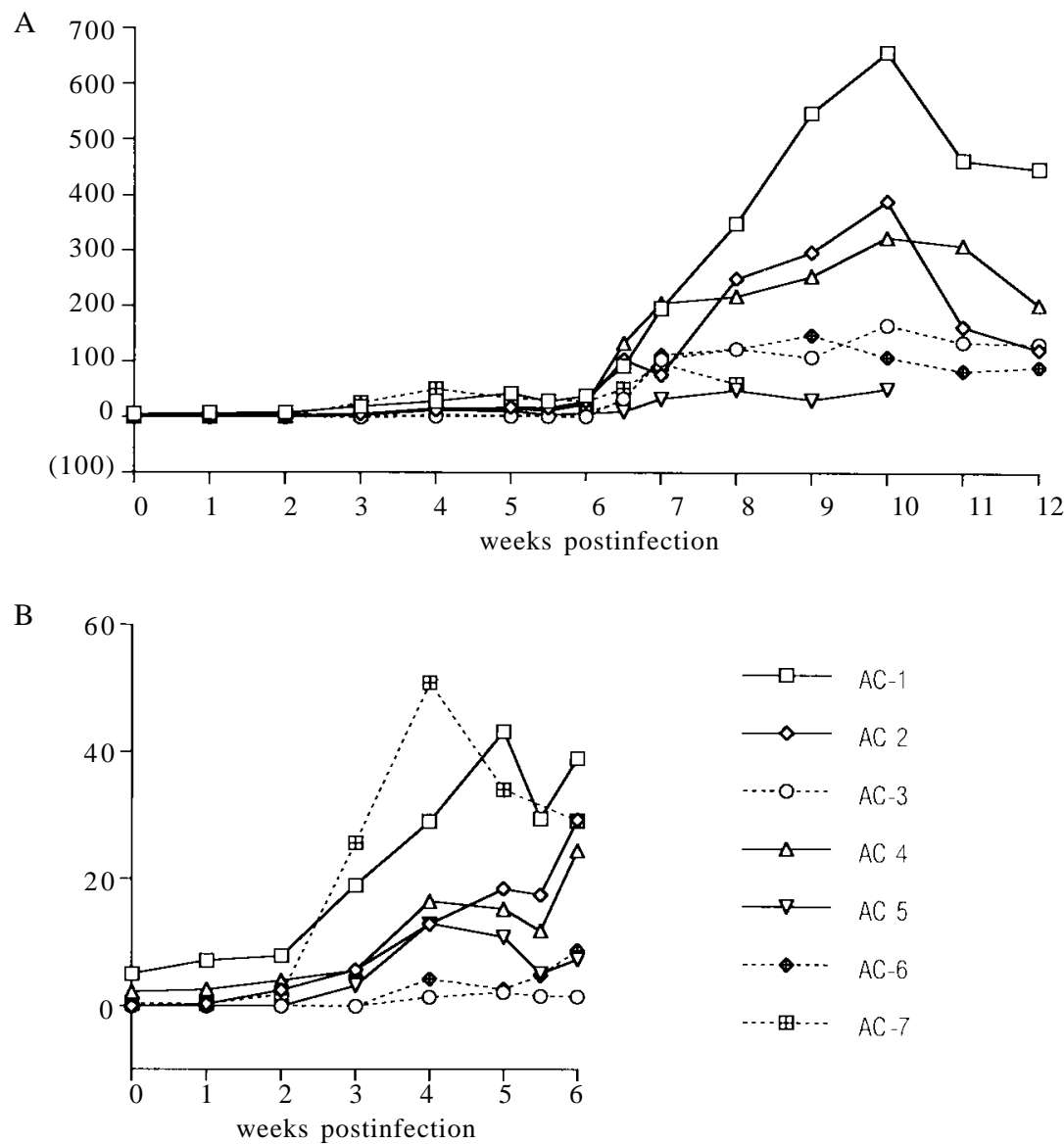

Fig. 1: antibody reactivity of sera from baboons acutely infected with Schistosoma mansoni to the S. mansoni adult worm microsomal antigen (MAMA), from 0 to 12 weeks postinfection. Antibody reactivity was measured in an ELISA assay, by comparison to a standard curve generated by dilutions of a positive sera pool, and expressed as units/ $\mu \mathrm{l}$. A: an overview of the pattern of response; B: a magnification of the early antibody response, from 0 to 6 weeks postinfection.

tion enzyme fragment that is 3511 bases in length. The insert sizes of the four selected clones, B4, A40, A47, and A27, varied from 1150 to 2006 bp (Fig. 2).

We took advantage of the different 5' ends of our cDNA clones and did some preliminary B cell epitope mapping. Using the $C$. parvum clone as the negative background, the four Smhsp70 clones were examined for antibody reactivity with a panel of baboon $S$. mansoni infection sera (Table). An example of the results from the immunoenzymatic plaque expression assay is shown in Fig. 3. The longest clone, B4, proved to be the most reactive, and the shortest clone, A27, the least reactive one.
Clone A40 showed a reactivity pattern identical to clone B4. Clone A47 was less reactive than clones B4 and A40 and was more reactive than clone A27. Of the twelve $S$. mansoni infected baboons only one, a chronically infected animal CR-4, lacked antibody reactivity with all of the clones. The preinfection sera from the twelve baboons lacked antibody reactivity with all of the clones.

Serial bleeds of one acutely infected baboon, AC-5, were tested in the immunoenzymatic plaque expression assay to determine the pattern of antibody development (Table). With this baboon, the pattern of antibody development corresponds to the overall antibody reactivity of

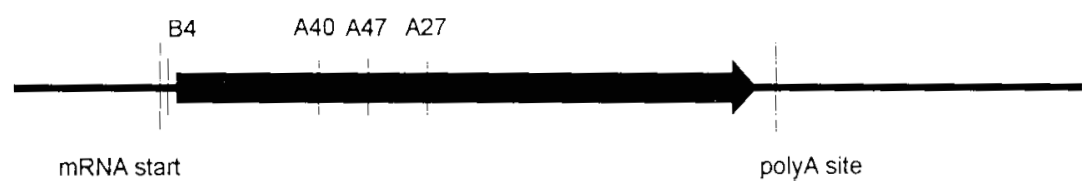

Fig. 2: the Schistosoma mansoni hsp70 gene. The coding sequence is indicated by the broad arrow and runs from base 533 to base 2446 . The mRNA begins at base 478 and ends at base 2509, the poly A site. The location of the 5' ends of our four hsp70 cDNA clones is shown. Clone B4 begins at base 503, 30 bases upstream of the translational start codon. Clones A40, A47, and A27 begin within the mRNA at bases 1001,1163 , and 1358 respectively. 
TABLE

Summary of the antibody reactivity of Schistosoma mansoni hsp70 clones; B4, A40, A47, and A27; in the plaque expression screening assay. Antibody reactivity was assessed with three groups of $S$. mansoni infected baboon sera. The first group contains eight, $\mathrm{AC}-1$ through $\mathrm{AC}-8$, acute infection sera obtained at 12 weeks postinfection. The second group contains four, CR-1 through CR-4, chronic infection sera obtained 5 to 10 years postinfection. The third group contains serial samples from one acutely infected baboon, AC-5. The antibody reactivity of the negative clone, Cryptosporidium parvum elongation factor 1-alpha, alone, is shown in the last column

\begin{tabular}{lccccc}
\hline & \multicolumn{5}{c}{ Clones } \\
\cline { 2 - 6 } Baboon sera & B4 & A40 & A47 & A27 & Negative \\
\hline AC-1 & + & + & $+/-$ & - & - \\
AC-2 & + & + & + & + & - \\
AC-3 & + & + & + & $+/-$ & - \\
AC-4 & + & + & $+/-$ & - & - \\
AC-5 & + & + & + & + & - \\
AC-6 & + & + & + & + & - \\
AC-7 & + & + & + & + & - \\
AC-8 & + & + & $+/-$ & - & - \\
CR-1 & + & + & + & + & - \\
CR-2 & + & + & + & + & - \\
CR-3 & + & + & $+/-$ & - & - \\
CR-4 & - & - & - & - & - \\
AC-5 1st wk pi & - & - & - & - & - \\
5th wk pi & - & - & - & - & - \\
6th wk pi & + & + & - & - & - \\
7th wk pi & + & + & $+/-$ & - & - \\
8th wk pi & + & + & + & + & - \\
9th wk pi & + & + & + & + & - \\
10th wk pi & + & + & + & + & - \\
11th wk pi & + & + & + & + & - \\
\hline
\end{tabular}

(+): reactive; (-): nonreactive; (+/-): variable result from assay to assay, sometimes weakly positive and sometimes negative; wk: week; pi: postinfection

the clones. Antibodies to clones B4 and A40 were first evident at 6 weeks postinfection. A weakly positive reaction to clone A47 was seen at week 7 and at week 8, all clones were reactive.

\section{DISCUSSION}

We were interested in identifying $S$. mansoni proteins potentially useful for detection of antibody in an early phase of acute infection. It is known that Smhsp70 induces a dominant antibody response in human and animals with both acute and chronic $S$. mansoni infection, and also in mice vaccinated with irradiated cercariae (Hedstrom et al. 1987, Moser et al. 1990). Our data showed that Smhsp70 could elicit a very early antibody response in the course of infection, at 6 weeks post infection; also, in three out of four cases, the antibody response was maintained in chronic infections.

An alignment of the Smhsp70 with Homo sapiens hsp70 have shown relatively few amino acid differences between the schistosome protein and the mammalian homologue, these differences being concentrated mainly on the Cterminal portion (Hedstron et al.1988, Newport et al. 1988, Neumann et al. 1992). In spite of this, the immune response to Smhsp70 appears to be specific. Healthy individuals and also patients with other helminthiasis, like clonorchiasis, schistosomiasis mekongi, loaiasis, bancroftian filariasis or cysticercosis did not react positively with the Smhsp70 (Newport et al. 1988). S. japonicum (Sj) hsp70 also showed an immunodominant response in schistosomiasis japonicum patients. Crossreactivity with the $S$. mansoni homologous antigen was not observed, suggesting some degree of species specificity (Hedstrom et al. 1988). The antibody response to our Smhsp70 clones, as observed in the $S$. mansoni infected baboons, seemed to be specific, since antibodies to hsp70 were not detected in any of the preinfection sera. So, considering the highly conserved nature of the members of this protein family, it seemed that the antibodies to hsp70 in schistosome infections are likely to be directed toward non-conserved regions of the protein.

The immunodominance of the hsp70 observed in different infections might reflect the relative abundance of this group of proteins, which are normally produced in higher amounts in response to a variety of stress conditions. Hsp70 was shown to be one of the first proteins synthesized by the transforming schistosomulae (Yukenberg et al. 1987). Analysis of RNA from different developmental stages of $S$. mansoni demonstrated expression of hsp70 in miracidia, sporocyst and adult worm, but not in cercaria. There is some evidence indicating that stage-specific expression of hsp70 is regulated not only by stress, but also by a developmental program. Cercaria/ schistosomula transformation can be considered as a physiological stress, involving increases of temperature and salt concentration, but these conditions alone were not sufficient to promote the enhancement of hsp70 expression. The loss of the cercarial tail was an obligatory event for hsp70 expression (Neumann et al. 1993). Its expression as soon as the cercaria have penetrated the skin and transformed in schistosomula may explain the early antibody response to hsp70 we observed in baboons, confirming results obtained by Hedstrom et al. (1987) in mice.

Smhsp70 seems to continue stimulating immune response in the chronic phase of infection, as shown by the detection of antibodies to this protein in three baboons with long lasting infection. The only chronically infected baboon (CR-4) that did not show antibody reactivity to the Smhsp70 clones was one that also showed very low antibody levels when tested by ELISA with total worm and egg antigens. The other three chronically infected baboons (CR-1, CR-2, CR-3) had high levels of antibodies against worm and egg antigens detected by ELISA (unpublished observations). So, the lack of response to hsp70 in this one baboon may be related more to the immune status of this baboon.

Moser et al. (1990) evaluated the diagnostic potential of two recombinant proteins corresponding to 121 and 230 amino acids of the C-terminal portion of the Smhsp70. They concluded that hsp70 was of limited value in the 


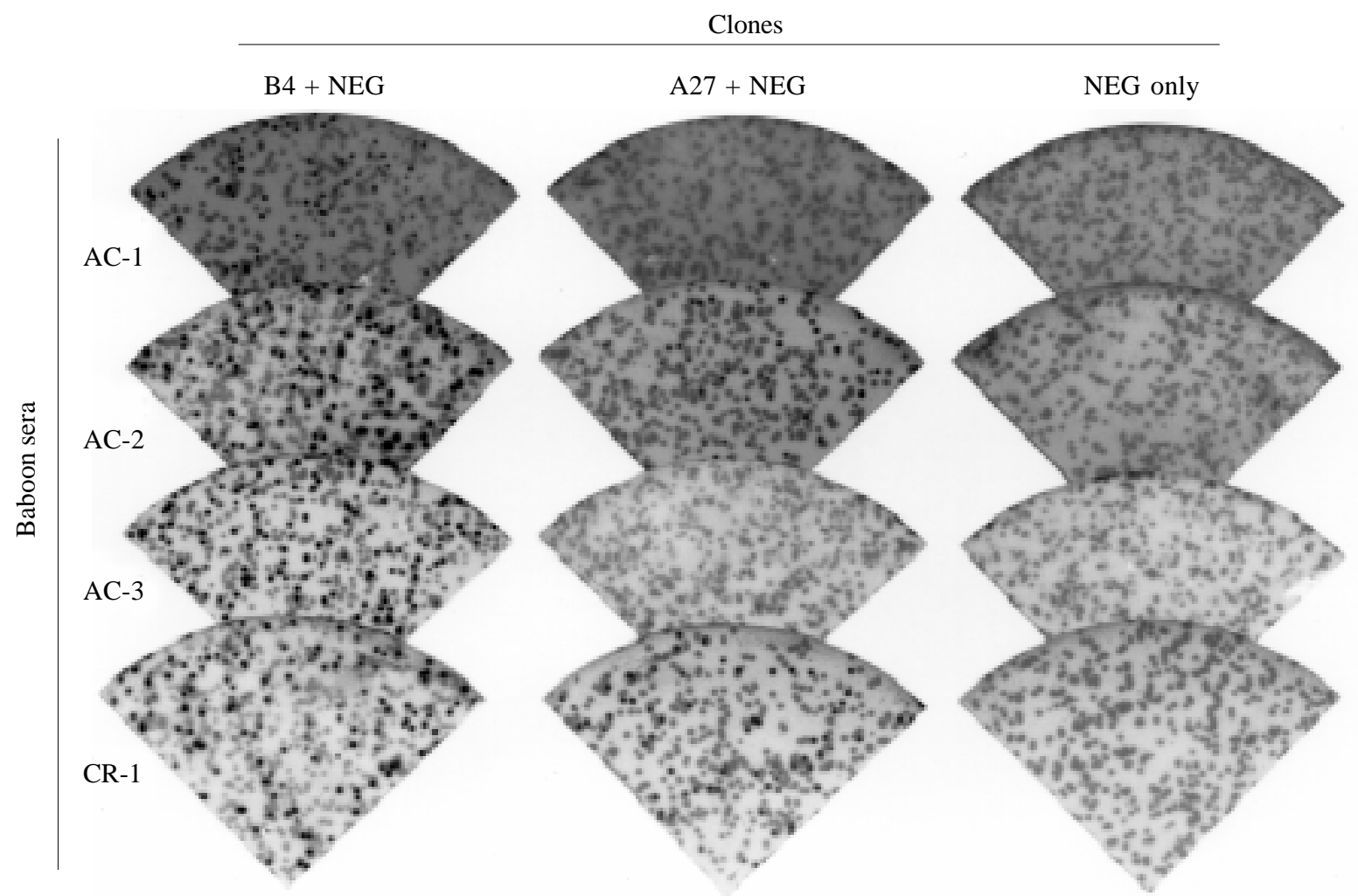

Fig. 3: antibody reactivity of Schistosoma mansoni hsp70 clones, B4 and A27, in the plaque expression screening assay, with sera from three baboons acutely infected (AC-1, AC-2, AC-3) and one baboon chronically infected with S. mansoni (CR-1). The hsp70 clones are plated with a negative clone background. The antibody reactivity of the negative clone, Cryptosporidium parvum elongation factor 1-alpha, alone is also shown.

diagnosis of schistosomiasis, because their recombinant proteins were not able to detect antibodies in patients with known recent infections. Their results confirmed that $\mathrm{Sm}$ and $\mathrm{Sj} \mathrm{hsp} 70$ are antigenicaly distinct, but cross-reactive antibodies were observed in some individuals with schistosomiasis haematobium and also in a small but significant number of patients with other parasitic infections. Our results showing strong reactivity of the baboon $S$. mansoni infected sera with the full-length recombinant protein (clone B4), and poor reactivity of the shorter clone (A27), can partially explain these results. Thus, it can be suggested that although a B-cell epitope must be present within the most immunogenic and hypervariable $\mathrm{C}$-terminal portion of the protein (Davenport et al. 1992, Wallace et al. 1992, Amorim et al. 1996), other antigenic determinants in the $\mathrm{N}$-terminal portion must contribute to the humoral immune response to hsp70. According to our data, epitopes that account for the greater reactivity of clones B4 and A40, as compared to A27, are between the sequences of clones A40 and A27. Of course, it is important to note that secondary structure and not just primary sequence may be involved. Our observations are in agreement with the results obtained by Quijada et al. (1996) that tested a recombinant $L$. infantum hsp70 with canine viscero-cutaneous leishmaniasis. They showed that while
$100 \%$ of the infected dog sera recognized the complete recombinant protein, only $30 \%$ of those sera recognized the 20 carboxyl-terminal amino acids of the protein.

Other authors (Andrade et al. 1992, Wallace et al. 1992, Arora et al. 1995, Quijada et al. 1996) have also demonstrated the immunodominance of hsp70 in patients with leishmaniasis, however conflicting data regarding to the cross reactivity of the leishmania hsp70 were presented. Epitope mapping studies carried out on hsp70 from different origins have demonstrated multiple antigenic determinants distributed along the protein in both conserved and non-conserved regions (Davenport et al. 1992, Wallace et al. 1992, Requena et al. 1993, Amorim et al. 1996). Since there are no other studies related to the variable antibody response against different segments of Smhsp70, the data presented in this paper can indicate the necessity of further studies to identify the cross reactive and specific epitopes of this protein. These studies will be important in order to determine the real value of hsp70 as an early diagnostic tool for schistosomiasis.

\section{ACKNOWLEDGMENT}

To Dr Jeffrey W Priest for the gift of the negative clone, Cryptosporidium parvum elongation factor 1-alpha in the vector lambda Uni-Zap XR. 


\section{REFERENCES}

Amorim AG, Carrington M, Miles MA, Barker DC, de Almeida M 1996. Identification of the C-terminal region of $70 \mathrm{kDa}$ heat shock protein from Leishmania (Viannia) braziliensis as a target for the humoral immune response. Cell Stress Chaperones 1: 177-187.

Andrade CR, Kirchhoff LV, Donelson JE, Otsu K 1992. Recombinant Leishmania hsp90 and hsp70 are recognized by sera from visceral leishmaniasis patients but not Chagas' disease patients. J Clin Microbiol 30: 330-335.

Arora SK, Melby PC, Sehgal S 1995. Lack of serological specificity of recombinant heat shock protein of Leishmania donovani. Immun Cell Biol 73: 446-451.

Bergquist NR 1995. Schistosomiasis vaccine development: approaches and prospects. Mem Inst Oswaldo Cruz 90: 221227.

Chitsulo L, Engels D, Montresor A, Savioli L 2000. The global status of schistosomiasis and its control. Acta Trop 77: 4151.

Davenport MP, McKenzie KR, Basten A, Britton WJ 1992. The variable C-terminal region of the Mycobacterium leprae 70-kilodalton heat shock protein is the target for humoral immune responses. Infect Immun 60: 1170-1177.

Davis A 2000. London School of Hygiene and Tropical Medicine Meeting at Keppel Street, London, 12 April 2000. The Professor Gerald Webbe Memorial Lecture: global control of schistosomiasis. Trans R Trop Med Hyg 94: 609-615.

Gibodat M, Bergquist NR 2000. Post-transmission schistosomiasis: a new agenda. Acta Trop 77: 3-7.

Hamilton JV, Klinkert M, Doenhoff MJ 1998. Diagnosis of schistosomiasis: antibody detection, with notes on parasitological and antigen detection methods. Parasitology 117 (Suppl.): S41-57.

Hancock K, Tsang VCW 1986. Development and optimization of the FAST-ELISA for detecting antibodies to Schistosoma mansoni. J Immunol Meth 92: 167-176.

Hedstrom R, Culpepper J, Harrison RA, Agabian N, Newport G 1987. A major immunogen in Schistosoma mansoni infections is homologous to the heat-shock protein hsp70. $J$ Exp Med 165: 1430-1435.

Hedstrom R, Culpepper J, Schinski V, Agabian N, Newport G 1988. Schistosome heat-shock proteins are immunologically distinct host-like antigens. Mol Biochem Parasitol 29: 275282.

Kakeya H, Udono H, Maesaki S, Sasaki E, Kawamura S, Hossain MA, Yamamoto Y, Sawai T, Fukuda M, Mitsutake K, Miyazaki Y, Tomono K, Tashiro T, Nakayama E, Kohno S 1999. Heat shock protein 70 (hsp70) as a major target of the antibody response in patients with pulmonary cryptococcosis. Clin Exp Immunol 115: 485-490.
Kanamura HY, Hancock K 1997. Rapid characterization of $S$. mansoni expression library clones of potential interest. Rev Inst Med Trop São Paulo 39: 355-357.

Kaufmann SHE 1990. Heat shock proteins and the immune response. Immunol Today 11: 129-136.

Moseley P 2000. Stress proteins and the immune response. Immunopharmacology 48: 299-302.

Moser D, Doumbo O, Klinkert MQ 1990. The humoral response to heat shock protein 70 in human and murine schistosomiasis mansoni. Parasite Immunol 12: 341-352.

Neumann S, Ziv E, Lantner F, Schechter I 1992. Cloning and sequencing of an hsp70 gene of Schistosoma mansoni. Mol Biochem Parasitol 56: 357-360.

Neumann S, Ziv E, Lantner F, Schechter I 1993. Regulation of hsp70 gene expression during the life cycle of the parasitic helminth Schistosoma mansoni. Eur J Biochem 212: 589596.

Newport GR, Hedstrom RC, Kallestad J, Tarr P, Klebanoff S, Agabian N 1988. Identification, molecular cloning, and expression of a schistosome antigen displaying diagnostic potential. Am J Trop Med Hyg 38: 540-546.

Oliveira-Ferreira J, Banic DM, Santos F, Ferreira-da-Cruz MF, Dubois P, Daniel-Ribeiro CT 1999. Cellular and antibody responses to the Plasmodium falciparum heat shock protein Pf72/HSP70 during and after acute malaria in individuals from an endemic area of Brazil. Acta Trop 73: 1-10.

Quijada L, Requeña JM, Soto M, Alonso C 1996. During canine viscero-cutaneous leishmaniasis the anti-hsp 70 antibodies are specifically elicited by the parasite protein. Parasitology 112: 277-284.

Rico AI, Angel SO, Alonso C, Requeña JM 1999. Immunostimulatory properties of the Leishmania infantum heat shock proteins HSP70 and HSP83. Mol Immunol 36: 11311139.

Requeña JM, Soto M, Guzman F, Maekelt A, Noya O, Patarroyo ME, Alonso C 1993. Mapping of antigenic determinants of the T. cruzi hsp70 in chagasic and healthy individuals. Mol Immunol 30: 1115-1121.

Sambrook J, Fritsch EF, Maniatis T 1989. Molecular Cloning: A Laboratory Manual, 2nd ed., Cold Spring Harbor Laboratory Press, Cold Spring Harbor, New York, p. 12.11-12.20.

Wallace GR, Ball AE, MacFarlane J, el Safi SH, Miles MA, Kelly JM 1992. Mapping of a visceral leishmaniasis-specific immunodominant B-cell epitope of Leishmania donovani hsp70. Infect Immun 60: 2688-2693.

Yuckenberg PD, Poupin F, Mansour TE 1987. Schistosoma mansoni: protein composition and synthesis during early development; evidence for early synthesis of heat schok proteins. Exp Parasitol 63: 301-311.

WHO - World Health Organization 2001. Schistosomiasis and soil-transmitted helminth infections. Wkly Epid Rec 76: 73-76. 